\title{
Agricultural research, or a new bioweapon system?
}

\author{
R. G. Reeves ${ }^{1}$, S. Voeneky², D. Caetano-Anollés ${ }^{1}$, F. Beck르, C. Boëte ${ }^{3}$ \\ ${ }^{1}$ Department of Evolutionary Genetics, Max Planck Institute for Evolutionary Biology, Plön, Germany. \\ ${ }^{2}$ Institute of International Law and Ethics of Law, University of Freiburg, Germany. \\ ${ }^{3}$ ISEM, Univ Montpellier, CNRS, EPHE, IRD, Montpellier, France. \\ Email: reeves@evolbio.mpg.de; svoeneky@jura.uni-freiburg.de
}

Agricultural genetic technologies typically achieve their agronomic aims by introducing laboratorygenerated modifications into target species' chromosomes. However, the speed and flexibility of this approach are limited, because modified chromosomes must be vertically inherited from one generation to the next. In an effort to remove this limitation, an ongoing research program funded by the U.S. Defense Advanced Research Projects Agency (DARPA) aims to disperse infectious genetically modified viruses that have been engineered to edit crop chromosomes directly in fields. This is genetic engineering through horizontal transfer, as opposed to vertical inheritance. The regulatory, biological, economic, and societal implications of dispersing such horizontal environmental genetic alteration agents (HEGAAs) into ecosystems are profound. Further, this program stipulates that the means of delivery of these viral HEGAAs into the environment should be insect-based dispersion (1). In the context of the stated aims of the DARPA program, it is our opinion that the knowledge to be gained from this program appears very limited in its capacity to enhance U.S. agriculture or respond to national emergencies (in either the short or long term). Furthermore, there has been an absence of adequate discussion regarding the major practical and regulatory impediments toward realizing the projected agricultural benefits. As a result, the program may be widely perceived as an effort to develop biological agents for hostile purposes and their means of delivery, which-if true-would constitute a breach of the Biological Weapons Convention (BWC).

Announced by DARPA in November 2016 (1), the Insect Allies program is reportedly backed by more than $\$ 27$ million in awarded research contracts (2-4). In July 2017, first of three consortia announced that they had been awarded a contract from DARPA to develop systems for insect dispersion of genetically modified viruses (2-4). These are contracts for completion of a 4-year work plan (1) that will culminate in large-scale greenhouse demonstrations of the fully functional insect-dispersed HEGAA approach. Maize and tomato plants are reportedly being used in current experiments (2-4), while dispersal insect species mentioned include leafhoppers, whiteflies, and aphids $(3,5)$.

Since its public inception, the Insect Allies program has almost exclusively been presented as a means for farmers to address routine agricultural concerns (e.g., drought, frost, flooding, salinity, herbicides, and disease) $(2-4,6)$. Despite a 2-year time frame for the generation of a functional proof-of-principle system in greenhouses (1), there has been little public explanation of how developments arising from conducting the work plan of the Insect Allies program might be applied to achieve the agricultural benefits highlighted by the funder or the researchers involved.

HEGAA Regulatory Pathways?

Although the program work plan does not fully prescribe the nature of the horizontal environmental "genetic alteration agent," it does stipulate the viral expression of $\geq 3$ transgenes to result in a gain-offunction phenotype in crop plants (1). The means by which these gains of function might be achieved remain to be established by the successful applicants, but the simplest route is most likely by editing genes on plant chromosomes (3-6).

Currently, the most obvious candidate for the HEGAAs (1) is a CRISPR system engineered to be part of a virus. Such an approach would target specific plant genes by modifying chromosomes, the alteration of which could increase plant resilience to environmental challenges or herbicides. The end effect of this system would be the use of a genetically modified virus to perform gene editing of susceptible crops in already-planted fields wherever the virus-carrying insects dispersed. It is conceivable that gains of function in the crop could be achieved by the expression of transgenes that were unable to modify the genome of the crop plant. However, numerous statements made by DARPA and involved researchers indicate that a core part of all the consortia's work programs likely includes plant chromosomal editing by means of virally encoded CRISPR proteins (4-6).

Press releases issued by DARPA and the lead institutions of the three consortia $(2-4,6)$ motivate the research goals exclusively or predominantly by references to routine use in agriculture, e.g., in relation to drought, frost, flooding, herbicides, salinity, or disease. This claim necessarily relies on the 
assumption that a plausible regulatory pathway exists by which possibly transgenic virus-infected (and potentially genetically altered) food crops could enter national or international markets (4). Even where there was obviously an established regulatory path for such routine use, it would still seem essential to clearly detail it; not to do so would risk undermining the repeatedly stated agricultural aims of the program (2-5). Yet there is no discussion at all in these press releases of regulation-a topic that is central to the question of intent. This omission is all the more striking given that it is likely that all current regulatory systems across the globe would require profound changes to accommodate even an occasional use of HEGAA technology (see the table).

Additionally, it is virtually certain that any concerns expressed by seed producers about how their facilities could be protected would receive considerable attention from regulators, farmers, and governments (see the table). Equally notable is the absence of any public discussion on how measures to ensure the coexistence of different farming practices, often mandated by law, could withstand the use of HEGAA technology.

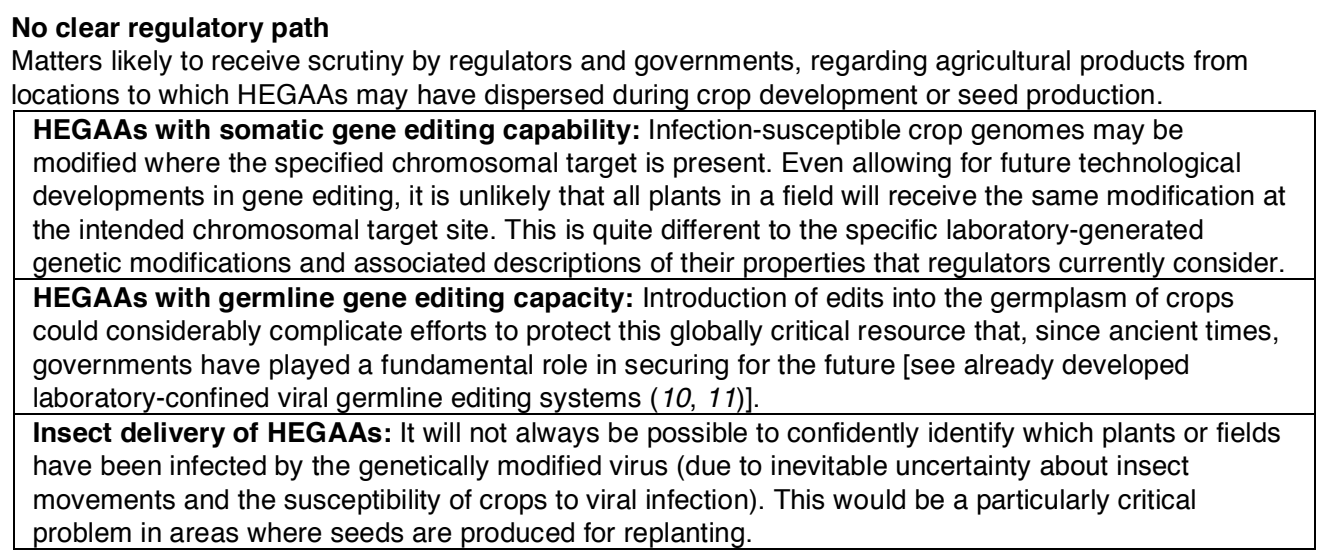

\section{Insect Dispersion and Intent}

Although the proposition of HEGAA technology is in itself radical, it is DARPA's mandate of using an insect-based means of delivery combined with HEGAAs that sets Insect Allies apart from all other programs. This is because it further increases incompatibilities with current farming practices, as well as the global regulation and trading of bioengineered food products, beyond the already substantial challenges stemming from the HEGAA component (see the table).

It would presumably have been possible for the DARPA work program to have proposed the development of HEGAAs to be deployed by using agricultural spraying equipment (7) without the involvement of insects. Crucially, all of the prominently hypothesized benefits to routine peacetime agriculture could probably be realized through the spraying of HEGAAs. It is therefore reasonable to ask, why mandate insect-based dispersion at all? The sole justification that has been put forward in any public document is that overhead spraying of HEGAAs would require infrastructure that is not available to all farmers $(1,6,8)$. Although this could conceivably provide a rather weak financial-efficiency justification for farmers in developing nations, it is hardly plausible when applied to the vast majority of crop farmers in the United States.

Although routine agricultural use is prominently presented in most documents as the motivation for the Insect Allies program, a secondary motivation is briefly acknowledged in some-namely its use as a defensive response to unspecified "threats introduced by state or nonstate actors" $(6,8)$. Only one of the three academic consortia makes clear reference to defensive or emergency applications in its press release (4). However, it is hard to imagine that U.S. farmers would not be ensured access to overhead spraying equipment (most likely via military and civilian aircraft) in the eventuality of such man-made threats. Furthermore, spraying capacity can be much more rapidly scaled-up than the mass production of insects, certainly within the time frame of a crop-growing season. In this respect, it is notable that the vast majority of frontline emergency measures to control insect pests for both agriculture and health continue to rely on spraying, even for pest species where control measures based on the release of live insects have been developed (e.g., sterile males). This is likely a reflection of the difficulty of rapidly scaling-up insect production and distribution systems.

If the DARPA program had proposed to enhance national overhead aerial spraying capacities, rather than stipulate the usage of insects as the means of delivery, it would be hard to argue that this would not result in a much greater enhancement of the United States' capacity to respond to hypothetical emergencies. Consequently, it is our opinion that until DARPA provides suitably robust explanations for 
the necessity of mandating insect dispersion in routine agricultural or emergency applications, Insect Allies risks being widely perceived as an attempt to develop a means of delivering HEGAAs for offensive purposes.

\section{Broader International Context}

The importance of perceived intent is of critical consideration in regard to allowing and encouraging scientific research for peaceful purposes while obeying international treaties that prohibit the use and development of certain weapons.

For example, the 1976 UN Convention on Environmental Modification Techniques states that it "shall not hinder the use of environmental modification techniques for peaceful purposes." However, the oftenunstated personal or institutional motives of research groups and funding bodies cannot be the sole basis upon which the wisdom of research programs is assessed. The 1972 BWC avoids relying solely on intent, insofar as it prohibits the development of biological agents "of types and in quantities that have no justification for prophylactic, protective or other peaceful purposes." Hence, a party engaging in the development of biological agents for which a hostile-use case is plausible (or even obvious, in the case of the Insect Allies program) must present acceptable explanations that its research is only serving peaceful purposes.

In addition, the BWC states that each State Party "undertakes never in any circumstances to develop, produce, stockpile or otherwise acquire or retain...weapons, equipment or means of delivery designed to use such agents or toxins for hostile purposes or in armed conflict." We therefore argue that the clause prohibiting the development of the "means of delivery" is likely to prove of particular relevance to the perceived intent of the Insect Allies program. In 2012, State Parties to the BWC expressly agreed that "certain developments in science and technology have the potential for use contrary to the provisions of the Convention now or in the future. These developments include, inter alia,...to develop novel means of delivering biological agents and toxins." (9).

Whether or not a chromosomal editing system (e.g., CRISPR) is ultimately used to achieve DARPA's stipulated aims, easy simplifications (and not elaborations) of the described work program could be used to generate a new class of biological weapon. For example, it is mandated that released insects must be subject to "conditional lethal safeguard(s)" whereby no released insects survive longer than 2 weeks. This is presumably to limit the dispersal of the HEGAAs (1). However, any program that would simply release insects without taking steps to implement some form of conditional lethality would have the effect of increasing the dispersal of HEGAAs into the environment.

HEGAA weapons could be extremely transmissible to susceptible crop species, particularly where insects were used as the means of delivery. Chromosomal editing would be targetable to particular crop varieties dependent on their genome sequence (presumably those varieties not grown by the deploying parties) (see the figure). nonspecialist scientists and policy-makers, even though it is anticipated that key development milestones should be achieved within the next year (1). Should this be accepted as the global norm for funding projects that enable such potentially hazardous directions of research, the best practices and rules, which have contributed to keeping our world largely free from the use of devastating biological weapons for over 60 years, may be seriously undermined.

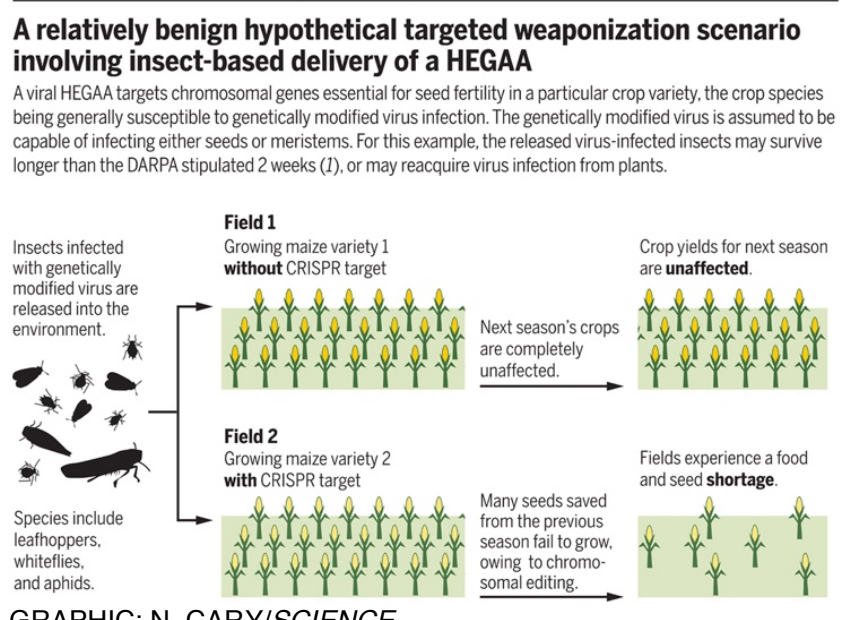

Possibly as a consequence of the program's contract-based funding process, we are unaware of any publicly available assessments of the ethical, trade, biosafety, or international biosecurity implications 
that would normally accompany such a globally important program of work (it is likely that congressional committees generated such assessments, but they are not in the public record). As a result, awareness of HEGAAs and insect-based means of their delivery is currently very low among

\section{Future Information Hazard}

There is likely to be widespread agreement with the sentiment that even legitimate biosecurity concerns should not undermine basic research and the development of biotechnologies for the good of humankind (Article $\mathrm{X}$ of the BWC). Unfortunately, the extent to which scientific developments will ultimately prove beneficial or harmful is not always straightforward to divine.

To be clear, it is not our contention that the Insect Allies program is ill-conceived simply because it is a military-funded program. Nor would we accept the assertion that the program is less problematic because it has been somewhat transparently initiated with academics. In our view, the program is primarily a bad idea because obvious simplifications of the work plan with already-existing technology $(10,11)$ can generate predictable and fast acting weapons, along with their means of delivery, capable of threatening virtually any crop species (see the figure).

Two of the three consortia funded by DARPA have publicly identified the target species for their experiments as maize - a crop upon which hundreds of millions of people rely for their basic nutritional needs, mainly in Latin America and Africa. Despite the contentious nature of what DARPA was proposing, the agency explicitly discounted funding projects that would target plant species with limited implications for global food security [specifically tobacco or Arabidopsis (1)]. This reflects the applied nature of DARPA funding, which aims toward making real-world impacts, primarily in the defense and security arenas. Although DARPA does "not anticipate applying publication restrictions" to results generated by the three research consortia [section 2.2 of (1)], one could argue that this may need to be reconsidered to avoid the proliferation of what may be seen as preliminary instruction manuals on how to develop offensive HEGAA programs, directed in the first instance against maize.

\section{Blurred Lines}

It is worth restating that weapons programs are often driven by perceptions of competitors' activities. Thus, it can be argued that participants in programs that operate close to the blurred lines dividing peacetime and wartime applications are obligated to project robust and plausible motivations for their work. It could, of course, be the case that DARPA is responding to specific intelligence about another state's objectives with, for example, its recent \$100 million programs incorporating Gene Drive (12) and Ecological Niche Preference Engineering (13). However, the mere announcement of the Insect Allies program, with its presented justifications, may motivate other countries to develop their own capabilities in this arena-indeed, it may have already done so (14). This will largely occur independently of whether or not the DARPA program is ultimately scientifically successful, or whether any of the results are made public as planned.

Reversal of funding for this DARPA project by the U.S. Congress [as has happened in the past with DARPA programs that had not received sufficient real-world consideration (15)] would not in itself close the particular Pandora's box that HEGAAs or their insect dispersal may represent. Nonetheless, there is a compelling argument that nowhere has bold leadership for the benefit of humankind been more internationally reciprocated than in the control of the use, development, or stockpiling of biological weapons.

\section{References and Notes}

«Broad Agency Announcement Insect Allies, Biological Technologies Office, HR001117S0002, 1 November 2016;

www.fbo.gov/utils/view?id=40638c9e7d45ed8310f9d4f4671b4a7b.

ABTI receives DARPA "Insect Allies" Award. EurekAlert! (2017); www.eurekalert.org/pub_releases/2017-07/bti-brd072717.php.Google Scholar

4 Penn State team receives $\$ 7 \mathrm{M}$ award to enlist insects as allies for food security (2017); http://news.psu.edu/story/495037/2017/11/20/research/penn-state-

team-receives-7m-award-enlist-insects-allies-food.

"Ohio State scientists make plant virus system "turn on its head" with insect research (2017); www.thelantern.com/2017/12/ohio-state-scientists-to-make-

plant-virus-system-turn-on-its-head-with-insect-research/.

IInsect Allies: How the Enemies of Corn May Someday Save It (2017); https://cals.ncsu.edu/news/insect-allies-how-the-enemies-of-corn-may-somedaysave-it/.

4DARPA Enlists Insects to Protect Agricultural Food Supply \& Commodity Crops. R\&D Mag. (2016); www.rdmag.com/news/2016/10/darpa-enlists-insectsprotect-agricultural-food-supply-commodity-crops.Google Scholar

$4 \mathrm{~J}$. S. Cory et al., Nature 370, 138 (1994). CrossRefWeb of ScienceGoogle Scholar

Dr. Blake Bextine, Insect Allies; www.darpa.mil/program/insect-allies.

Report of the Meeting of the States Parties to the Convention on the Prohibition of the Development, Production and Stockpiling of Bacteriological (Biological)

and Toxin Weapons and on Their Destruction, Geneva (2012); http://undocs.org/en/BWC/MSP/2012/5.

\&Z. Ali et al., Mol. Plant. 8, 1288 (2015).CrossRefGoogle Scholar

AK. Musiychuk et al., Influenza Other Respir. Viruses. 1, 19 (2007).CrossRefPubMedGoogle Scholar

4DARPA:Safe Genes; www.darpa.mil/program/safe-genes.

DARPA:Young Faculty Awards 2017; www.darpa.mil/work-with-us/for-universities/young-faculty-award.

AUnited States of America, Strengthening of the BWC, Meeting of the States Parties to the Convention on the Prohibition of the Development, Production and

Stockpiling of Bacteriological (Biological) and Toxin Weapons and on Their Destruction, Geneva (2018); http://undocs.org/en/BWC/MSP/2018/MX.5/WP.3

4J. W. Schoen, Pentagon kills 'terror futures market.' msnbc.com (2003); www.nbcnews.com/id/3072985/t/pentagon-kills-terror-futures-market/. Acknowledgments: R.G.R. and S.V. contributed equally to this work. 